\title{
LIMITED COMMON GROUND, UNLIMITED COMMUNICATIVE SUCCESS: AN EXPERIMENTAL STUDY INTO LINGUA RECEPTIVA USING ESTONIAN AND RUSSIAN
}

\author{
Daria Bahtina-Jantsikene \\ University of Helsinki
}

\section{Ad Backus}

Tilburg University

\begin{abstract}
Previous research on Estonian-Russian interaction suggests that lingua receptiva (LaRa), or Receptive Multilingualism, has the potential to create, boost or restore common ground, or mutual understanding, in situations where common ground is jeopardized. This mode is characterized by the simultaneous use of multiple languages as interlocutors each speak their mother tongue and count on the receptive skills of the other. Alignment in the LaRa mode was tested in a series of experiments. The use of various meta-communicative strategies provided insight into the mechanisms behind LaRa. Their distribution was influenced somewhat by L2 proficiency and exposure to multilingual communicative situations. Interestingly, higher L2 knowledge was not a prerequisite for success. Moreover, it was the composition of the dyad rather than the characteristics of the individuals that had predictive power regarding communicative success.
\end{abstract}

Keywords: lingua receptiva, meta-communicative devices, L2 proficiency, multilingualism, common ground, Estonian, Russian

\section{Introduction}

Communicative success can be conceptualized as the degree to which interlocutors manage to reach common ground (Clark 1996). At the most atomic level of a communicative exchange, two alternating turns between two people engaged in a dialogue, a speaker aims 
to get a message across and the hearer is supposed to understand that message. If the latter indeed 'gets it', the two partners may be said to have reached maximal common ground, as they both now know the same thing. In designing a message, a speaker takes into account an educated guess about the state of knowledge the hearer possesses before the exchange. This is easier if there is already a lot of common ground to begin with. In such cases, the hearer will understand most things without problem, including not just the words and grammatical patterns that the speaker uses, but also what's behind all the stylistic choices, the nods and head turns, the intonation patterns, etc. This is why communication with friends or family members about familiar topics in familiar settings tends to be so fast and effortless. At the other extreme are unfamiliar communicative settings. Lack of familiarity can result from at least two sources. The interlocutors might not know each other well, or the communicative task may be unfamiliar. Lack of familiarity produces stressful situations characterized by low degrees of common ground. Communicative success needs effort.

This article reports on an experimental study in which pre-existing common ground was kept small, to see how people would handle this situation and what strategies they would use to nevertheless ensure successful communication. Specifically, language choice was manipulated. Bilingual speakers of Estonian and Russian were instructed to only use their mother tongue in communication with mother tongue speakers of the other language, a pattern known as Lingua Receptiva (LaRa) or Receptive Multilingualism. This way of communicating, while conventional in a number of multilingual settings around the world, was virtually unknown to the participants. Some of the results were somewhat surprising, as it turned out that limitations in pre-existing common ground sometimes had a beneficial effect for the conversational task, essentially because it induced people to help each other more.

The first section will discuss the role of common ground in communication in general and the way in which it was kept small in the 
present study. The subsequent sections present the methodology and the results, focusing on the abovementioned surprises. The final section explores the implications of these findings for future studies.

\section{Common ground}

The importance of 'common ground' for communication was emphasized by Clark (1996), as the central concept in his theory of communication (and also taken up by Pickering, Garrod 2004, Tomasello 2008, Croft 2009, and others). In communication, people strive towards maximizing the common ground between them, and the inherent puzzle of communication is how this is achieved given that two interlocutors can never know for sure how much common ground they have between them.

Common ground is greater the more familiar the communicative setting is, and familiarity is high when interlocutors know each other well, and the setting is like many other settings the interlocutors have experienced in the past. Often, these two sources of familiarity go together, for example when a group of close friends engage in small talk on their weekly night out. However, close friends too may sometimes engage in a conversation type they don't experience together often, for example when one of them suddenly has to explain a dramatic development at his job (e.g. lay-offs) despite the organizational features of his workplace having never before been a topic of conversation in the group. Other examples would include an academic professor telling his/her non-academic cousin about his research when he/she has never done such a thing before, or colleagues running into each other in town, and realizing they have so far only talked about task-related issues at work and never exchanged small talk.

In psycholinguistics, common ground is often conceptualized as alignment between speakers. Research tends to focus on 'deep' cognitive processes that are beyond the conscious attention of language users (Pickering, Garrod 2004). Primary evidence for unconscious or 
automatic processing is the existence of priming effects in dialogue. If Speaker A uses a particular word or construction, the chances that Interlocutor B will use it too increase, without any evidence that this was because of a conscious act of imitation or because Speaker A somehow urged him to. Alignment can exist at various levels, and Pickering, Garrod (2004) argue for a system in which alignment on one level induces alignment at 'higher' levels, so that priming at lexical and syntactic levels contributes to speaker and hearer mentally representing the same proposition (which they call alignment of 'situation models'). At the same time, however, alignment can also be achieved through more conscious efforts, for example by actually checking whether the hearer has understood what was said. Also, if communication partners are interested in harmony, understanding and conviviality, they might imitate and accommodate to each other for social reasons. A desire for cementing the bond between speaker and hearer increases the chance of mutual understanding because it increases the wish to align. The present study focuses on such conscious ways of facilitating alignment. The participants in our study had to overtly attempt to bring about alignment at the propositional level, and one of our prime objectives was to investigate the means by which they did that.

We particularly focus on how interlocutors overcame the limitation of not being allowed to use the other's first language, and how they used 'meta-communicative devices' (henceforth: 'MCDs') to do this. MCDs and similar concepts have been discussed in various schools in pragmatics, interactional sociolinguistics and conversational analysis, and have consequently been referred to with various terms (e.g. 'hearer-oriented strategies' in Functional Pragmatics, cf. Rehbein et al. 2012). Non-automatic alignment is achieved on the basis of conscious moves that speakers and hearers make in conversation. As these serve to regulate the communication itself, rather than convey referential content, they are 'meta'-communicative. Examples include explicit negotiation about the communicative task at hand, or explicit attention to how a particular word should be 
understood in the current context. Further details will be given in the 'Method' section.

\section{The current study}

Common ground is greatest when people who know each other well engage in conversational settings that are routine. In order to study how people manage to build common ground in unfamiliar conversational settings, an experiment was designed in which participants engaged in a communicative task, a so-called 'maze task'. The task was characterized by four features designed to lower the level of familiarity.

First, the interlocutors did not know each other. As a result, they could not rely on the historically built communicative common ground that close friends share. Not having extensive communicative history makes it harder to understand each other's intentions. Second, all participants had a different native language from that of their assigned interlocutor (though they knew the language of the other as an L2, to varying degrees). If your interlocutor speaks the same language as you, you can rely on extensive linguistic common ground, especially if you also share similar social and geographical backgrounds. If you are paired with an L2 speaker, on the other hand, you cannot know as well whether the words and grammatical constructions you employ are known by him or her. Third, as mentioned, they each had to use their own native language during the communicative task they carried out. As participants will not be familiar with this way of communicating, they could not rely extensively on communicative routines that were familiar to them. $\mathrm{LaRa}$ is generally assumed to be possible as long as the languages are mutually intelligible, as in much of Scandinavia. However, there is a logical second possibility: speakers can also understand each other if they have learned each other's languages. In that case, the native languages of the two interlocutors do not have to be mutually intelligible. This is what applies to our case, as our participants 
are bilingual in Russian and Estonian (see the section 'Methodology'). One important consequence when LaRa is dependent on mastery of a second language ('Acquired LaRa') is that you can never be sure how much the partner understands. Few people learn a second language so perfectly that they can pass for native speakers, so one always has to work with less than perfect understanding. Finally, the dialogue participants had to interact through Skype, without video, rather than face-to-face. This meant there was relatively poor physical common ground. Nonverbal features such as gestures and body language, which people normally use in meaningful ways in faceto-face communication, could not be used. We were interested in seeing how pairs of participants would try to reach common ground in this less than ideal situation.

The study employed a maze task (cf. Pickering, Garrod 2004). As experimental designs go, maze tasks lead to reasonably natural conversation while allowing for considerable experimental control over the language use that participants will produce. We were particularly interested in whether participants would compensate for the lack of common ground by an increased use of MCDs.

\section{Methodology}

The study we report on had participants carry out a maze game in pairs, through the medium of Skype.

\section{Participants}

As it was important to keep pre-existing common ground relatively small, we paired interlocutors who did not know each other, except for the pilot run, in which participants were recruited from the same company. The members of each pair were native speakers of different languages, one Estonian and one Russian, except for ten monolingual 'control' pairs. As all participants were residents of Estonia, they were bilingual to some extent, but varied in the degree to which 
they knew the other participant's $\mathrm{L} 1^{1}$. The participants were preselected so that there were comparable numbers of dyads with specific L2 proficiency combinations (both advanced L2-speakers, both limited, mixed).

The 96 participants were grouped into 38 pairs that communicated in the LaRa mode (the 'experimental group'), five pairs that communicated in Russian only and five that communicated in Estonian only (the 'control groups'). Even though the latter two groups communicated in their mother tongue, they were part of the same bilingual Russian-Estonian speech community as the participants in the experimental condition. All participants were told that their interlocutor had at least some command of their L1.

All participants were adults (age range 22-56), two thirds were female, and all had an educational background that included higher education (some were undergraduates and the rest had completed their studies). They were paid for their participation and were informed that the study looked into multilingual practices, but no further details about its purpose were provided.

\section{Materials and Procedure}

As mentioned, the study was designed to limit pre-existing common ground between participants. That was one reason why they were required to communicate with someone who was speaking a language that was an L2 for them. LaRa has been studied either from a historical perspective (e.g., Braunmüller 2007) or in naturalistic settings (e.g. Beerkens 2010). This study was the first that explored

\footnotetext{
1 Due to historical developments in Estonia over the past 50 years, the respective L2 proficiency is highly asymmetrical across the two speech communities as well as across generations. According to the Estonian Integration Monitoring report from 2015, younger generations of Russian-speakers are increasingly more proficient in Estonian whereas it is mostly older generations of native Estonian speakers who have an active command of Russian as L2 (yet, the majority claims at least passive knowledge of Russian regardless of the age group).
} 
this communication mode in an experimentally controlled environment (see Blees et al 2014 for a similar study). In addition, the study was set up in such a way that people were expected to put effort into maximizing the resources they have in their repertoire. The maze game is not easy: participants need to coordinate their efforts, and the task is virtually impossible to complete without the use of MCDs, needed as they are for checking whether the participants are both on the same wavelength. In order to gain enough information on participants' linguistic and sociolinguistic abilities, they were given a sociolinguistic questionnaire and a second language proficiency test to complete.

The sociolinguistic questionnaire was presented in the mother tongue of the participant and covered self-reported language proficiencies, the extent to which the participant had experience with multilingual situations, and the participant's attitudes towards Estonian and Russian speakers. Self-assessed L2 proficiency was used to pair participants into dyads with specific combinations.

L2 proficiency was further tested through a cloze-test using a set of L2 texts. We opted for a flexible grading system which allowed the inclusion of the partial knowledge that is crucial in LaRa dialogues: responses that included orthographic mistakes or stylistically poor choices were not discarded, but lowered the scores.

The pairs had to jointly conduct the maze task through telephone-like conversations on Skype. In such tasks, participants have to find their way around on a fictional street plan; in our case they had to find each other's location on a very abstract map designed specially to reduce the level of familiarity. It excluded landmarks, place names or any other references that would make the task simple. One extra measure that was taken to increase the difficulty of the task was that there were small differences between the fictional maps of each speaker in a dyad. It was assumed that resolving this task would require active discussion and efforts to reach mutual understanding. The time allocated for completing the task was limited to ten minutes and success was defined as complete, partial or 
zero based on whether they managed to locate both, one or no position on the map.

All conversations were transcribed with EXMARaLDA free software tools (Schmidt, Wörner 2009) and then annotated using a unified coding system. The parameters marked during annotation, as far as relevant to the present article, included time to completion, level of success, number of utterances per dyad and per interlocutor, the number of the meta-communicative devices used and their distribution, and the total percentage of interaction that was in the LaRa mode. The results were subjected to statistical analysis, and are reported in full in Bahtina-Jantsikene (2013).

In this paper, we first provide some of these results and then focus on the use of MCDs. While similar categories and terms exist in related fields, we based our classification on the traditions in functional pragmatics and psycholinguistic alignment. We defined four hierarchically ordered meta-communicative devices: MCD1 is any form of explicit negotiation about the communicative task at hand, aiming for achieving common ground about the goal of communication, e.g. 'what are we doing here?' Assuming this higher-order alignment has been established, MCD2 is used to make sure the specifics of the current communicative task are shared, e.g. 'do you see $\mathrm{X}$ on your map?' in the case of our maze task. Even when overall and specific goals are known by both interlocutors, they may not possess sufficient knowledge of all linguistic forms that are used (including codeswitching). MCD3 helps create this knowledge by focusing the other's attention on specific linguistic forms (e.g. 'what does that word mean?'). Finally, MCD4 can feature any of the above but has the overall goal of explicitly checking for shared understanding ('confirmation checks', e.g. 'do you follow?').

The hypothesis was that there would be a trade-off between degree of pre-existing common ground and the use of MCDs: the less one can expect the hearer to understand what one is saying (i.e. the lower the hearer's proficiency in your native language), the more extensive the use of MCDs would be. One might also expect 
particular types of MCDs to be especially relevant in particular constellations of linguistic proficiency of the dyad, and perhaps also be sensitive to other features, such as the dyad's experience with multilingual communication. However, we also remained open to the idea that MCDs are used simply for phatic reasons, since it is also conducive to communicative success if the communication partners establish positive rapport.

\section{Results}

The general results were published in Bahtina-Jantsikene (2013) and will only briefly be summarized here, so see also Bahtina et al. (2013). The analysis here will focus mostly on the use of MCDs, in line with our research question about the degree to which participants in a communicative exchange help each other reach understanding when common ground can be presumed to be small. The first sub-section summarizes the main findings of the whole study; the second section deals with MCDs and their distribution.

\section{Main findings}

We ran a series of tests aimed at understanding the relation between L2 composition of the dyad and their success rate, also taking into account the degree to which participants said they had experience with multilingual communicative contexts. Table 1 contains a selection of results: we report here only significant differences. For that reason, not all dyad types are represented in this Table or in Table 2 in the next subsection. If for a particular dyad type (e.g. HL; i.e. one member with High and one with Low L2 proficiency) there was no significant result for a particular outcome (e.g. reaching zero success), it is not included. The results show a surprisingly low direct impact of high L2 proficiency; one would instead expect the higher degree of common ground that this entails to have a beneficial effect on communicative efficiency. Pairs in which both partners had high 
L2 proficiency were not faster than pairs in which L2 proficiency was distributed more unevenly. Perhaps even more surprising, the monolingual pairs (the 'control groups') were not statistically faster at completing the maze game than the bilingual pairs. These results suggest that the degree of pre-existing common ground is not necessarily predictive of communicative success, at least not when measured in terms of shared linguistic means. Advanced command of a second language did not guarantee mutual understanding. In fact, dyads with mixed L2 proficiencies had higher success rates on average.

Table I: Statistically significant effects of linguistic proficiency on speed and success rates $(\mathrm{HH}=$ dyad with high $\mathrm{L} 2$ proficiency levels; $\mathrm{HL}=$ dyad with mixed L2 proficiency levels)

\begin{tabular}{|c|c|}
\hline Measure of success & Differences that reach significance \\
\hline $\begin{array}{l}\text { Speed of finding points } \\
\text { on the map } \\
\text { (in seconds) }\end{array}$ & $\begin{array}{l}\text { HH dyads find Ist point on the map slower than } \\
\text { Monolingual pairs, } \\
t(24)=2.65, p=0.0 I \\
\text { HL dyads find one point fast, } \\
F(I, 36)=4.28, p=0.05 \\
\text { HL dyads find both points fast, } \\
F(I, 3 I)=5 . I I, p=0.03\end{array}$ \\
\hline $\begin{array}{l}\text { Level of success } \\
\text { (zero - partial - full) }\end{array}$ & $\begin{array}{l}\text { HL dyads reach partial rather than full success, } \\
F(I, 36)=4.05, p=0.05 \\
H L \text { dyads with higher Russian } L 2 \text { reach partial rather than } \\
\text { full success, } F(I, 36)=5.50, p=0.02 \\
\text { HL and } L L \text { dyads reach higher level of success, } \\
\text { Fisher's } 2 * 2, p=0.0 \text { I }\end{array}$ \\
\hline
\end{tabular}

LaRa dyads in which both interlocutors had higher L2 scores were significantly slower than their monolingual counterparts. This suggests that in this multilingual mode better command of the other's language can even be an obstacle. Completely against expectation, pairs in which one of the participants reported lower L2 proficiency corresponded with higher success rates and faster completion of the 
task. One explanation would be that the partner of a participant with low L2 skills may have been extra careful to make him- or herself understood. The latter point would be in accordance with the general expectation that whenever trouble is to be expected in communication people will try harder to establish common ground, for example by using more MCDs.

\section{MCD use}

Next, we look at the use of meta-communicative devices, specifically focusing on whether their use correlates with L2 proficiency and exposure to multilingual situations. One might expect that experienced multilingual communicators make judicious use of MCDs to enhance understanding. We also expected these MCDs to particularly be of the type where it is checked whether the interlocutor has understood ('confirmation checks'). The results show that this is indeed the case, but with a twist. Dyads in which both interlocutors had high L2 proficiency ('HH') and lots of experience with bilingual communication, showed significantly higher MCD use. Yet, in pairs with extensive experience with bilingual communication confirmation checks (MCD 4) were used much less. Further examination of the data revealed pronounced differences in the distribution of MCDs: L2 proficiency and degree of experience with multilingual situations affected the choice of MCD type. Table 2 illustrates the relevant results.

MCD use was highest for speakers who reported high exposure to the L2 and who were in pairs in which both speakers reported high L2 proficiency. This suggests that MCD use comes naturally to speakers who often communicate in bilingual settings. A likely explanation is that such speakers have learned through experience that intercultural communication is more successful if one uses MCDs. Interestingly, among the dyads that reached full success L1 Russian speakers with high exposure to L2 Estonian used MCDs significantly less often. These findings suggest that the impact of 
Table 2: Statistically significant effects of MCD use on task success and of background factors (L2 proficiency combination, experience with multilingual situations, and attitudes towards respective $L 2$ and its speakers) on MCD use

\begin{tabular}{|c|c|}
\hline MCD type & Distribution / Success level \\
\hline $\begin{array}{l}\text { Overall use } \\
\text { of MCDs }\end{array}$ & $\begin{array}{l}\text { HH dyads use more MCDs than } H L, r(I 6)=0.5 I, p=0.04 \\
\text { Subjects with LI Russian and high exposure to Estonian use fewer MCDs } \\
\text { and reach higher success, } r(16)=-0.54, p=0.03 \\
\text { Subjects with LI Estonian and positive attitude to Russian use fewer MCDs } \\
\text { and reach higher success, } r(16)=-0.60, p=0.01\end{array}$ \\
\hline MCDI & $\begin{array}{l}\text { Faster at finding the Ist point on the map, } r(26)=4 . \mathrm{I}, \mathrm{p}<0.05 \\
\text { Faster at finding the } 2 \text { nd point on the map, } r(23)=6.4, \mathrm{p}<0.0 \mathrm{I} \\
\text { Faster at finding both points on the map, } r(33)=3.5, \mathrm{p}<0.05 \\
\text { HL dyads with higher } \mathrm{L} 2 \text { exposure use more } \mathrm{MCD} \text {, } r(22)=0.49, \mathrm{p}=0.02 \\
\mathrm{HL} \text { dyads with subjects with LI Estonian and higher exposure to Russian } \\
\text { use more } \mathrm{MCDI}, \mathrm{r}(22)=0.64, \mathrm{p}<0.0 \mathrm{I}\end{array}$ \\
\hline MCD2 & $\begin{array}{l}\mathrm{HH} \text { dyads use more MCD2 than } \mathrm{HL}, \mathrm{F}(\mathrm{I}, 36)=5.83, \mathrm{p}=0.02 \\
\text { Subjects with } \mathrm{LI} \text { Estonian and high exposure to Russian use more MCD2 } \\
\text { and reach higher success, } \mathrm{r}(\mathrm{I} 6)=.49, \mathrm{p}=0.05\end{array}$ \\
\hline MCD3 & $\begin{array}{l}\text { Subjects with lower } L 2 \text { tend to use more } M C D 3, r(38)=-0.22, p=0.06 \\
\text { Subjects with } L I \text { Russian and lower } L 2 \text { tend to use more } M C D 3 \text {, } \\
r(38)=-0.3 \mathrm{I}, \mathrm{p}=0.06 \\
\mathrm{HH} \text { dyads with positive attitudes to respective } \mathrm{L} 2 \text { use more } M C D 3 \text {, } \\
r(\mathrm{I} 6)=0.53, p=0.04 \\
\text { HH dyads with subjects with } \mathrm{LI} \text { Russian and positive attitudes to } L 2 \\
\text { use more } M C D 3, r(I 6)=0.56, p=0.02\end{array}$ \\
\hline MCD4 & $\begin{array}{l}\mathrm{HL} \text { dyads with subjects with } \mathrm{LI} \text { Estonian and higher exposure to } \mathrm{L} 2 \\
\text { use fewer } M C D 4, r(22)=-0.50, p=0.02\end{array}$ \\
\hline $\begin{array}{l}\text { MCDs } \\
\text { in phases: } \\
\text { Before } \\
\text { finding first } \\
\text { point vs } \\
\text { after }\end{array}$ & $\begin{array}{l}\text { HH dyads use more MCDs in Phase I than } \mathrm{HL} \text { dyads, } \mathrm{F}(\mathrm{I}, 24)=7.25 \text {, } \\
\mathrm{p}=0.0 \mathrm{I} \\
\mathrm{HH} \text { dyads use more MCDs in Phase I than in Phase } 2, \mathrm{t}(9)=2.9 \mathrm{I}, \mathrm{p}=0.02 \\
\text { More MCDs in Phase } 2 \text { in full success dyads, } \mathrm{F}(\mathrm{I}, 24)=4.60, \mathrm{p}=0.4 \\
\text { More MCDs in Phase } \mathrm{I} \text { in slow dyads, } \mathrm{r}(26)=-0.82, \mathrm{p}<0.0 \mathrm{I} \\
\text { More MCDs in Phase } 2 \text { in fast dyads, } \mathrm{r}(26)=0.73, \mathrm{p}<0.0 \mathrm{I}\end{array}$ \\
\hline
\end{tabular}


exposure and L2 proficiency on task success and on MCD use is not straightforward (but see Discussion).

Using MCD1, i.e. conducting explicit negotiation about the goals of the communicative event, correlated positively with the speed of task completion. Extensive previous experience with bilingual communication appears to stimulate the use of this type of discourse technique. There was no correlation with L2 proficiency, however, only with amount of experience, and this holds especially for people whose $\mathrm{L} 1$ is Estonian.

MCD2 is a more difficult device to use, as it requires sophisticated vocabulary to check with the interlocutor whether the layout and orientation of the maps speaker and hearer have in front of them match. It was indeed used more by pairs that should have the least trouble understanding one another: pairs with higher L2 proficiencies ('HH'). More bilingual experience also corresponded with an increased use of this MCD type as did higher chance of success in the experiment, at least for Estonian L1 speakers.

While MCDs 1 and 2 are tightly related to the task at hand, and could be said to be triggered by its unfamiliar nature, the other two MCDs involve strategies people employ every day in conversations with friends, colleagues, officials and strangers. MCD3 was characterized as 'creating linguistic common ground', and includes all meta-talk as well as code-switching. Though it did not correlate with higher success rates in the task or with going faster through it, this device was used both for functional reasons (by less proficient L2 speakers) and presumably phatic ones (by participants with high L2 and self-reported positive attitude to the L2). What this suggests is that when speakers feel understanding is compromised by a perceived lack of sufficient L2 skills on the part of the interlocutor, MCD3 serves as a means to compensate. An example comes from a dyad with an Estonian speaker who was not so fluent in Russian. She informs her interlocutor about a break in communication (Est: 'ee... ma ei tea seda sõna vist', Eng: 'ehm... guess I do not know this word') and the Russian-speaking participant provides a translation 
of the key word (Est: 'ülesse', Eng: 'up'). Once the issue is resolved, they continue in their respective L1. Phatic use was in evidence when sufficient L2 knowledge made it safe to talk normally: one HH dyad upon reaching the point on the map that was manipulated to look different for the two interlocutors realized that they had misaligned and the Russian-speaking participant commented in Estonian 'juba mingi jama!' ('already some mess!'), before continuing in Russian, both of them laughing.

MCD4, characterized as a confirmation check, can be viewed as the simplest mechanism to restore understanding in challenging, unfamiliar situations. Little can be said about its use in the data, at least as far as correlations with background characteristics, speed and success rates are concerned. The only significant result suggests that this strategy is used less when pairs have higher experience with bilingual communication, especially in dyads with mixed L2 proficiency. This 'parroting' device could be assumed to compensate for a lack of (perceived) initial common ground, but it becomes less relevant when extensive multilingual experience provides more confidence in one's intercultural abilities.

As for development during the execution of the task, HL pairs, i.e. pairs in which one speaker had low L2 proficiency, tended to use more MCDs, especially MCD3, as the task progressed. In contrast, $\mathrm{HH}$ pairs tapered off their use of MCDs. Concerning the effect of MCD use on creating mutual understanding, dyads that continued using MCDs in the second phase, were remarkably better both in terms of task completion and speed in getting there.

\section{Discussion}

Perhaps the first result to highlight is that the LaRa mode of communication, despite participants' lack of experience with it, did not keep people from communicating meaningfully and successfully. It has its limitations, though, as can be seen by the trouble it caused pairs in which both members spoke the other language well. This is 
probably because in such situations few people will naturally choose to practice LaRa, unless they already have a communicative history with their interlocutor in which this mode has been established.

It is of course puzzling that pairs which reported low or even zero L2 proficiency still managed to communicate successfully in the LaRa mode. There are various possible explanations. Presumably, some participants underestimated their L2 proficiency. Second, it might be the case that the lexical and grammatical skills tested in standard proficiency tests, such as the cloze test we used, do not suffice. Communicative competence is the ability to interact, rather than just talk, but such skills were not tested. Finally, our experimental design may have induced some particular effects not readily found in daily life. First, the difficulty of the communicative task encouraged people with limited grammatical and lexical competence in L2 to make full use of other aspects of their communicative competence, including the judicious use of MCDs as speakers and elicitation of MCDs as hearers. There is some evidence in our data that pairs that could expect communicative trouble worked harder at avoiding it, while pairs who assumed they could rely on the high L2 proficiency of each partner, appeared to underestimate the need to attend to each other's degree of understanding. This interactive nature of competence also explains why the individual characteristics of the subjects - those of a speaker rather than of a dyad - had very little predictive power when we looked at overall success rates or at MCD use. Second, as one reviewer pointed out to us, the maze task constrains the sorts of meaning any utterance may be conveying. The circumscribed nature of the task in effect increases the common ground to higher levels than would be the case in ordinary communicative settings.

MCDs catch the moment where interlocutors interrupt or slow down the conversation to avoid possible misunderstanding. The question is what prompts speakers to make more or less extensive use of MCDs. The decision to use them needs to be interpreted as part of a larger complex of behavioral characteristics, including 
lessons from past experience, attitudes to interlocutor and language, the nature of the communicative task, amongst probably many other aspects. Past experience determines which discourse patterns are entrenched in a speaker's mind. Someone who has often been in intercultural communicative settings will have seen how problems with understanding emerge, have practiced ways of avoiding misunderstanding, and have developed ideas about what works and what does not. Experienced intercultural communicators are likely to have developed sophisticated insights into the common sense notion that you need to help interlocutors who don't speak your language well.

In multilingual settings, codeswitching often functions as a contextualization cue, assisting both speakers and hearers in elucidating pragmatic meaning. Often, it has the general phatic effect of cementing the bond of community among bilinguals. In the experimental setting, codeswitching was effectively banned, as speakers were supposed to speak their L1 throughout. Nevertheless, various instances were attested, and they often appeared to serve phatic functions. This use of codeswitching could be interpreted as a general MCD: it makes the conversation more pleasant because it allows speakers to meet the other halfway. At the same time, specific cases of codeswitching may be used simply to clear up a very local problem of understanding, for example if a particular L1 word is not understood by the partner and the speaker therefore inserts the L2 equivalent. On an even more general level, codeswitching is just a special case of 'languaging': using whatever resources that seem to get the communicative job done.

When it comes to other factors of influence, extensive experience with bilingual situations and having a positive attitude towards the L2 and its speakers repeatedly showed their impact. These features seemed to help speakers overcome the disadvantages of low L2 proficiency and in some cases helped such pairs in achieving higher levels of success in the maze task. In this respect, communicative competence, in addition to having its source in linguistic knowledge 
and interactional experience, also includes sociolinguistic components and is affected by interpersonal and intercultural attitudes.

The degree to which MCD use evolved during the Skype conversations suggests a close connection between MCD selection and proficiency. Recall that pairs in which one speaker had low L2 proficiency tended to use more MCD3 as the task progressed, while $\mathrm{HH}$ pairs reduced their use of MCDs, and the one they used most was MCD2, which indeed requires higher linguistic skill. This suggests that the pairs who could expect communication breakdowns tried harder to actively construct common ground. Obviously, if your proficiency is higher, you are also better able to use MCDs, but the data also show that they are very useful tools exactly when proficiency is low. Interestingly, such extensive use of MCDs also seems to lead to improved results for task completion, perhaps as a by-product of the conscious need to increase common ground.

\section{Future research}

The study answered some questions but elicited new ones. A number of factors were found to impact communication in unexpected ways. However, further exploration of these requires more than a post hoc analysis. MCD use was found to differ depending on the linguistic composition of the pairs and participants' experience with bilingual situations in general. In currently on-going follow-up research, we systematically vary several types of familiarity: L2 proficiency, degree of multilingual experience, and experience with particular communicative settings. We also vary the communicative tasks, since some of the results we obtained may have been caused by particular features of the maze task.

In the new study interlocutors familiar with each other engage with each other in situations they know well since they have experienced them many times before, but also in contexts that do not resemble their typical communicative constellations. Other factors that are manipulated include the extent of experience that 
communicators have with the skills needed to conduct the conversation: especially whether they are native speakers, how good their L2 skills are, and to what extent they have experience with multilingual settings. The data reported on in this paper suggest that when familiarity is low, judicious use of meta-communicative devices becomes more important. This may also hold for related strategies not investigated yet, such as the use of communicative routines, phonological reduction, and joint construction of discourse. It will also be investigated what the effect is of the presence of a third participant. An uninformed third interlocutor instructed to jump in whenever there is a sign of communication breakdown is used to analyze how (potential) misunderstanding is detected, prevented or fixed in a multi-party conversation. The results of this kind of research may be relevant in the development of training modules for intercultural communication and other linguistic and interactional constellations that are prone to miscommunication due to lack of familiarity.

\section{REFERENCES}

Bahtina-Jantsikene, Daria 2013. Mind Your Languages: Lingua Receptiva in Estonian-Russian Communication. LOT dissertation series 338. Utrecht University.

Bahtina, Daria; ten Thije Jan D.; and Wijnen, Frank 2013. Combining cognitive and interactive approaches to Lingua Receptiva. International Journal of Multilingualism 10 (2), 159-180.

Beerkens, Roos 2010. Receptive Multilingualism as a Language Mode in the Dutch-German Border Area. Münster: Waxmann Verlag.

Blees, Gerda; Mak, Willem; and ten Thije, Jan (2014). English as a lingua franca versus lingua receptiva in problem-solving conversations between Dutch and German students. Applied Linguistics Review, 5 (1), 173-193.

Braunmüller, Kurt 2007. Receptive Multilingualism in Northern Europe in the Middle Ages: A description of a scenario. In J. ten Thije and L. Zeevaert, eds. Receptive Multilingualism: Linguistic Analyses, Language Policies and Didactic Concepts, 25-49. Amsterdam: John Benjamins.

Clark, Herbert H. 1996. Using Language. Cambridge University Press. 
Croft, William 2009. Toward a Social Cognitive Linguistics. In V. Evans and S. Pourcel, eds. New Directions in Cognitive Linguistics, 395-420. Amsterdam: John Benjamins.

Estonian Integration Monitoring 2015. Carried out by the Institute of Baltic Studies, Tallinn University and Praxis Center for Policy Studies. Available at http://www.kul.ee/sites/kulminn/files/6peatykk.pdf

Pickering, Martin J.; and Simon Garrod 2004. Toward a mechanistic psychology of dialogue. Behavioral and Brain Sciences 27, 169-225.

Rehbein, J., ten Thije, J.D. and Verschik, A. (2012). Remarks on the Quintessence of Receptive Multilingualism. In ten Thije, J. D.; Rehbein, J. and Verschik, A. (Eds.) Lingua Receptiva. International Journal for Bilingualism 16 (3), 248-264.

Schmidt, Thomas; Wörner, Kai (2009). EXMARaLDA - Creating, sharing and analyzing spoken language corpora for pragmatic research. Pragmatics 19 (4), 565-582.

Tomasello, Michael 2008. Origins of Human Communication. MIT Press. 